\title{
Functional interpretation of ovarian cancer in correlation with
}

\section{ISGF3 expression pattern}

\author{
Qinglian Ma ${ }^{1,2}$, Wenjie Yan ${ }^{1}$, Jing Yang ${ }^{1}$, Haiyan Wang ${ }^{2}$, Weixiang Wang ${ }^{2}$, Minghui Dong ${ }^{2}$ \\ ${ }^{1}$ Reproductive Medical Center, Renmin Hospital of Wuhan University, Wuhan, P. R. China \\ ${ }^{2}$ Department of Obstetrics and Gynecology, Wuhan Red Cross Hospital, Wuhan, P. R. China
}

\begin{abstract}
Summary
Purpose: Interferons are frequently used as an agent in cancer therapy because they possess tumor suppressor activity. The aim of the present work is to investigate the therapeutic role of interferon stimulated gene factor 3 (ISGF3) in different pathological stages of ovarian cancer. Materials and Methods: Sprague Dawley rats were surgically operated along with carcinogen 7,12-Dimethylbenzanthracene (DMBA) to develop ovarian cancer. Histology was used to analyze the grade of ovarian cancer. Immunohistochemistry and Western blotting technique were used to understand the expression of CK7 and ISGF3 $\gamma$ expression. Results: The rat incubated with carcinogen for continuous 18 and 24 weeks were able to develop low and high-grade ovarian cancer respectively. Histologically, low-grade tumor showed more transitional malignant cells while high-grade ovarian cancer showed higher number of proliferative and clumpy cells. The expression of CK7 is constantly overexpressed as tumor pregressed with band intensity of 2.7 and 4.6-fold higher in low and high-grade ovarian cancer. In contrast, interestingly ISGF3 $\gamma$ showed 4.3-fold higher expression in low grade ovarian cancer, but limited with only 1.7-fold higher expression in high grade ovarian cancer. Conclusion: The present results concludes that higher expression of ISGF3 $\gamma$ in low-grade ovarian tumor is due to suppression of tumor development, but in absence of ISGF3 $\gamma$, expression of tumor development is uncontrolled.
\end{abstract}

Key words: ISGF3; Cytokeratin 7; DMBA; Ovarian cancer; Membrane accentuation.

\section{Introduction}

Ovarian cancer accounts for the fifth leading cancer among women population and it also emerges as a more lethal form of gynaecological cancer in developing countries $[1,2]$. Although there is much development in cancer surgical strategies as well as in the treatment procedures, the survival rate of cervical cancer patient has not improved to considerable level for the past 20 years [2]. Currently the studies show that five-year survival rate of an advanced stage patient is only below $29 \%$ and in $52 \%$ of cases in women above 60 years results in death [2]. Other challenges behind ovarian cancer are multidrug resistance and tumor recurrence $[3,4]$. Therefore, there is an urgent requirement for an early diagnosis and treatment of ovarian cancer at molecular level.

The tumor microenvironment which is composed of endothelial cells, fibroblast, extracellular matrix, and immune cells, which respond differently and thereby promote tissue remodeling, angiogenesis, and cell proliferation [5]. Although immune cells have the ability to recognize and kill the cancer cells in some circumstances, they favor tumor growth and metastasis [6, 7]. The cellular movement connected with cancer development is associated with inflammation-driven corrupted immune system [8]. The inflammatory response that develops in different stages of tumorigenesis alter genome stability, induce cellular proliferation, enhance epigenetic modification, as well as antiapop- totic cellular pathways [9].

Interferon stimulated gene factor 3 (ISGF3) is a multisubunit transcriptional factor that induces the expression of many interferon stimulated genes $[10,11]$. Functional form of ISGF3 is typically composed of two components, namely ISGF $3 \alpha$ and ISGF3 $\gamma$. STAT $1 \alpha(91 \mathrm{kDa})$ or STAT1 $\beta(84$ $\mathrm{kDa})$ along with STAT2 (113 kDa) form ISGF3 $\alpha$, which are activated by type I interferons that later bind with ISGF3 $\gamma$ to form a functional complex. The functional complex formed in the cytoplasm are translocated to nucleus and upregulate the transcription of interferon stimulated genes (insert reff here). In the present investigation, the expression of ISGF3 $\gamma$ in different pathological stages of ovarian cancer were analyzed.

\section{Materials and Methods}

Fifteen female Sprague Dawley rats of three months old were purchased. The animals were acclimatized in laboratory conditions for one week. The rats were divided into three equal groups $(\mathrm{n}=5)$ and maintained in individual wire cages at a temperature $\left(23 \pm 2{ }^{\circ} \mathrm{C}\right)$ with relative humidity $(55 \pm 60 \%)$. The mice were illuminated for 12 hours/day and fed ad libitium with pellet food and water. For induction of ovarian cancer, the mice were first anesthetized using intraperitoneal injection of ketamine hydrochloride $(100 \mathrm{mg} / \mathrm{kg})$. Following abdominal hair removal from surgical site. The left ovary was externalized
Eur. J. Gynaecol. Oncol. - ISSN: 0392-2936 XLI, n. 5, 2020

doi: $10.31083 /$ j.ejgo.2020.05.5320
This is an open access article under the CC BY-NC 4.0 license (https://creativecommons.org/licenses/by-nc/4.0/). 
using a small peritoneal incision. Silk suture (size 3.0) impregnated with carcinogen 7,12-Dimethylbenzanthracene (DMBA) was implanted for tumor initiation, as previously described [12] and the wound was closed using five sutures. Following surgery, the rats were carefully monitored each week for tumor development using palpation of left side abdomen. The rats were able to develop initial and advanced stages of ovarian cancer following 18 and 24 weeks of incubation, respectively. Following the incubation period the rats were sacrificed and ovarian samples were dissected for different experimental analyses. All animal handling and experimental procedure were previously approved by the Institutional ethical approval committee and extreme care was taken to minimize pain.

The normal and tumor samples from initial and advanced stage of ovarian cancer, rats were excised surgically and washed with distilled water. The tissues samples were fixed with $10 \%$ formalin solution for 72 hours at room temperature. Following fixation, the tissues were subjected to dehydration using gradual increase in isopropyl alcohol concentration. This was followed by incubation with Xylene for 45 minutes which acted as a clearing agent and aided in wax infiltration. Paraffin embedded wax was then subjected to $6-\mu \mathrm{m}$ thin size sectioning and further processed for visualization in different sections of the slide. Hematoxylin and Eosin staining was employed to stain the cytoplasm and nucleus, respectively. Slides were mounted using DPX without forming air bubbles and allowed to dry before prior examination.

The paraffin embedded tissues were subjected to $6-\mu \mathrm{m}$ thin sectioning and placed on a glass slide. Following dewaxing, the sections were immersed in the antigen retrieval solution ( $10 \mathrm{mM}$ sodium citrate buffer, $\mathrm{pH}$ 6) at $95^{\circ} \mathrm{C}$ for 15 minutes. The cellular endogenous peroxidase activity was neutralized for 20 minutes by treating the sections with 3\% $\mathrm{H}_{2} \mathrm{O}_{2}$ in freshly prepared methanol solution. After one hour of incubation in blocking serum the sections were incubated with primary antibody, either (anti-ISGF3G, HPA001862) or (anti-cytokeratin 7 antibody) for six hours at $4{ }^{\circ} \mathrm{C}$. Following washing with $1 \times$ PBS solution, the slides were incubated with suitable secondary antibody for two hours at room temperature. The slides were washed again to remove non-specific binding of antibody. The sections were developed with Diaminobenzidine (DAB) kit to achieve the chromogenic signals. The slides were counterstained with Hematoxylin and mounted using the DPX solution for visualization.

Normal and ovarian cancer tissue samples were homogenized in $2 \times$ sample buffer in ice cold condition. The samples were subjected to heat in a water bath for ten minutes, and after cooling the samples were stored at $-20{ }^{\circ} \mathrm{C}$. For Western blot analysis the protein samples were run on $10 \%$ SDS PAGE gel with the equal loading of protein samples $(60 \mu \mathrm{g})$ and transferred to PVDF membrane. After blocking with $4 \%$ BSA solution the membrane was incubated with primary antibody either (anti-ISGF3G, HPA001862) or (anti-cytokeratin 7 antibody) for six hours at $4{ }^{\circ} \mathrm{C}$. After washing with TBST solution for three times, the membranes were incubated with suitable secondary antibody (alkaline phosphatase conjugated) for another two hours at room temperature. Following washing step with TBST the membrane was developed using BCIP/NBT chromogenic substrate to obtain signals. The signals were quantified based on the band intensity and was documented.

The comparison of data obtained against different groups were analyzed using student $t$-test (SPSS Statistics v13.0) and expressed in mean $\pm \mathrm{SD}$. The $p$ value was considered significant when it was less than 0.05 .

\section{Results}

Using carcinogen DMBA and by following standard protocol as described in Materials and Methods, the rats successfully developed low and high-grade ovarian cancer at 18 and 24-week intervals respectively. These rats were subjected to histological sectioning in order to confirm the pathological stages. The normal ovarian tissue was composed of a solid mass of cells without any abnormal variations (Figure 1A). In addition to that we observed, small follicles and cells in the tissue appeared similar with monomorphic appearance (Figure 1A). The DMBA mediated ovarian cancer induced rats after 18 weeks showed transitional forms of cells with a low-grade benign form of malignant cells (Figure 1B). The cells were morphologically abnormal in size with clear large proliferative cells (Figure 1B). The rats incubated for 24 weeks showed a rigid form of cells with higher proliferative index (Figure 1C). The solid clumps of cells with invasive nature were growing rapidly to form high-grade malignant cells (Figure 1C).

Cytokeratin 7 (CK7) expression in ovarian tissue with strong staining are a positive sign of ovarian cancer $[13,14]$. The expression of CK7 in normal ovarian cancer was very limited (Figure 2A), while the characteristic nature of CK7 expression in low-grade ovarian cancer represented higher expression beneath the membrane than in the central cytoplasmic region (Figure 2B). However profound expression of CK7 was observed in high-grade ovarian cancer (Figure 2C) which indicated the severe nature of DMBA following 24 weeks of incubation.

Developmental characteristics of ovarian cancer in context to ISGF3 $\gamma$ expression was analyzed in normal, low, and high-grade ovarian cancer tissues. In the control ovarian tissue, ISGF3 $\gamma$ showed less positive cells (Figure 2D) but its distinctive expression was greatly enhanced in lowgrade ovarian tissue (Figure 2E). During cancer upgrading, ISGF3 $\gamma$ expression was further demarcated with a significant reduction pattern of expression in high-grade ovarian cancer (Figure 2F).

To determine the precise relative expression of $\mathrm{CK} 7$ and ISGF3 $\gamma$ in immunohistochemistry, Western blotting experiments were performed. The data showed that as abnormal proliferation of ovarian cells increased, there was a gradual upregulation of CK7 pattern (Figure 3). In depth observa- 
A

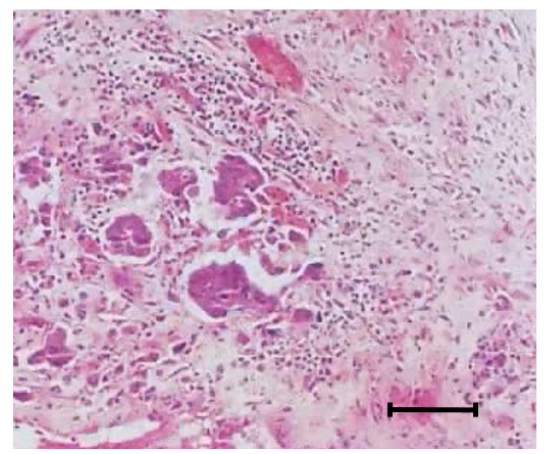

B

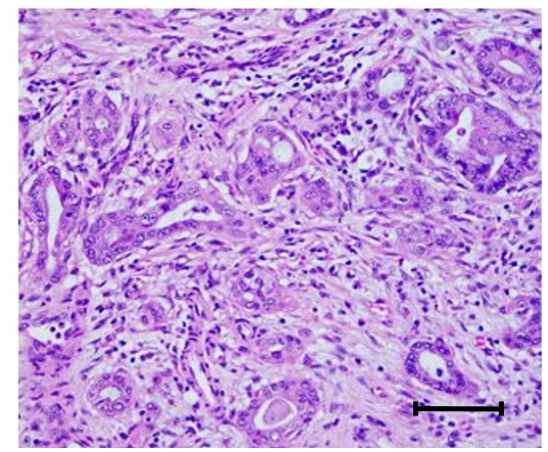

C

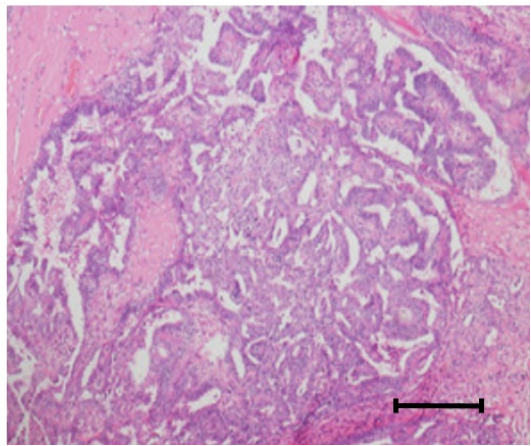

Figure 1. - Histological observation of ovarian cancer development. A. Histological sections of normal ovarian tissue without any abnormal morphological structure. B. Low-grade ovarian tissue with a transitional form of ovarian malignant cells, which begin to divide uncontrollably. C. High-grade ovarian cancer tissue with more proliferative cells along with clumping cells. Hematoxylin and Eosin stained. Scale bar $-\times 20$.

A

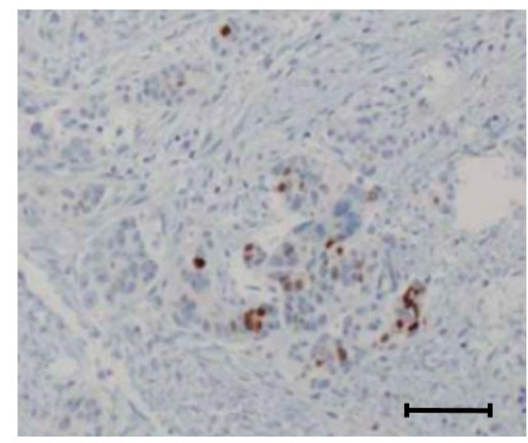

D

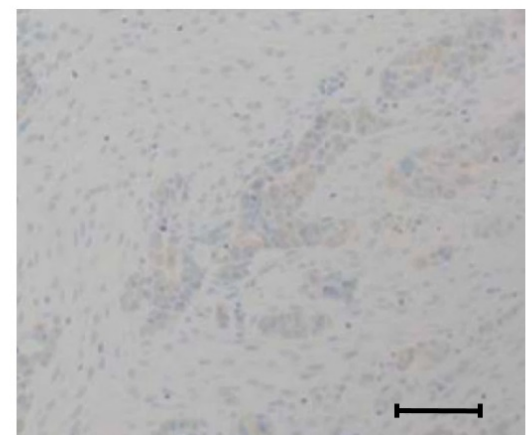

B

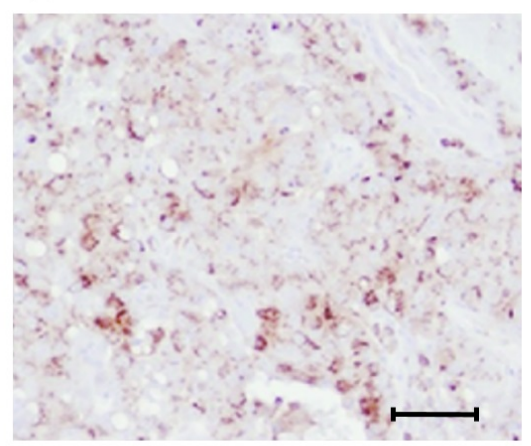

$\mathrm{E}$

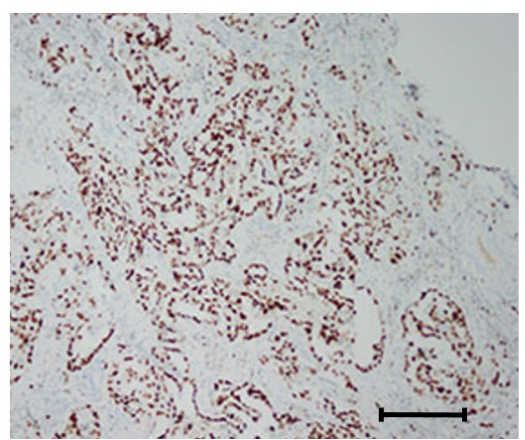

C

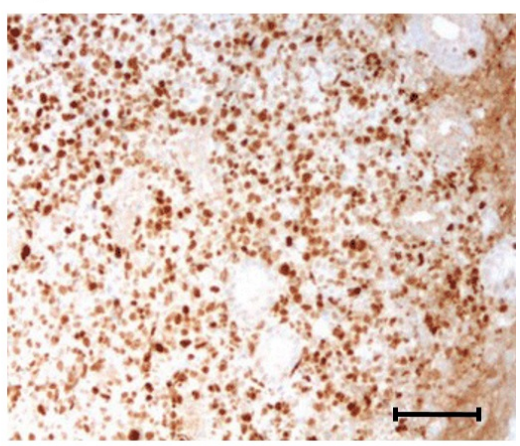

$\mathrm{F}$

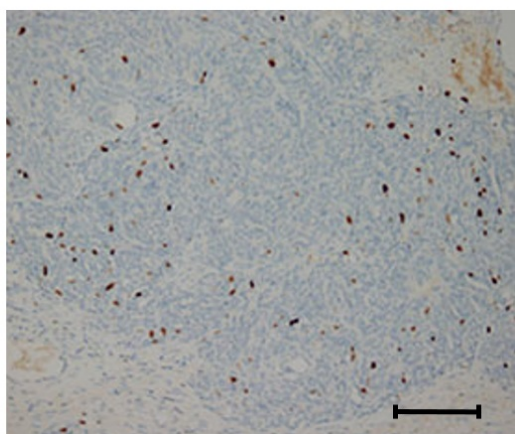

Figure 2. - Immunohistochemical expression of CK7 and ISGF3 $\gamma$ in different grade of ovarian cancer. A. Normal ovarian tissue with restricted expression of CK7. B. Low-grade ovarian cancer tissue with a marginal upregulated expression of CK7 with membrane accentuation. C. High-grade ovarian cancer tissue showing enhanced expression of CK7 with more cytoplasmic accentuation. D. Normal ovarian tissue showing very limited expression of ISGF3 $\gamma$. E. Upregulated expression of ISGF3 $\gamma$ in low-grade ovarian cancer tissue. F. High-grade ovarian cancer tissue showing downregulated expression of ISGF3 $\gamma$. Scale bar $-\times 20$.

tion against band intensity showed that the expression of CK7 was 2.7-fold higher in low-grade ovarian cancer when compared with control tissue. Similarly, as cancer progressed to high-grade, the band intensity represented 4.6fold expression than the control tissue. Also, the association of ISGF3 $\gamma$ expression in low-grade ovarian cancer was 4.3fold higher than control tissue, but it showed only a 1.7-fold more expression in high-grade ovarian cancer when compared with control tissue (Figure 3).

\section{Discussion}

Present study highlights the significant role of interferon stimulated gene factor 3 potentially important for tumor suppression in ovarian cancer. Generally, interferons show 


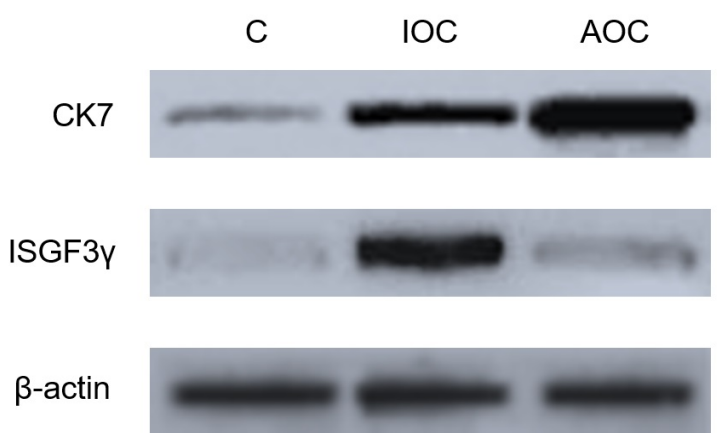

Figure 3. - Immunoblotting with anti-CK7 and anti-ISGF3 $\gamma$ antibody against low and high grade ovarian cancer. Lane 1: control ovarian tissue showing the expression pattern of CK7, ISGF3 $\gamma$, and $\beta$-actin. Lane 2: low grade ovarian tissue showing limited upregulation of CK7, increased upregulated expression of ISGF3 $\gamma$, and unchanged expression of $\beta$-actin. Lane 3: high grade ovarian tissue showing increased upregulation of CK7, downregulated expression of ISGF $3 \gamma$, and unaltered expression of $\beta$-actin. IOCInitial Ovarian Cancer; AOC-Advanced Ovarian Cancer.

anti-tumor effect which is regulated by interferon signaling regulating genes [15]. Previous studies with ISGF3 showed that its expression is associated with viral infection, DNA damage, and immunotherapies [16]. Recent investigation shows that ISGF3 is involved in tumor suppression of clear cell renal cell carcinoma (ccRCC) [17]. However, its role in ovarian cancer is not been explored so far. The present investigation mainly focused on understanding ISGF3 expression pattern in different pathological stages of ovarian cancer. As previously reported in clear cell renal cell carcinoma, the loss of HIF2a, KDM5C or PBRM in VHL-/cells are able to downregulate the expression of ISGF3 [17]. Also, studies show that the loss of BAP1 or SETD2 reduces the expression level of ISGF3 which confirms its expression are interrelated to each other [17]. Correlating with ovarian cancer, the higher and lower expression of ISGF3 in initial and advanced stage of ovarian cancer may be also link with the related proteins like HIF2a, KDM5C, PBRM, BAP1 or SETD2.

Typical characteristics of low and high-grade ovarian cancer was achieved through carcinogenic DMBA impregnation in the ovary and maintaining it for 18 and 24 weeks, respectively. The rats used in the experiment were not immune-compromised, but the authors used Sprague Dawley rats which are routinely used for oncology and immunological studies [18]. Parameters such as histology with increasing population of proliferation, along with abnormal cellular morphological structures, confirm the existence of low and high grade ovarian cancer. CK7 is a useful marker to identify ovarian cancer and helps differentiate various origins of ovarian cancer [19]. In the present investigation, the authors observed a significant increase in expression pattern of CK 7 following adverse condition of cancer development.

Interestingly, CK7 expression in low-grade ovarian can- cer is observed within inner cytoplasmic membrane, but upon tumor progression, the CK7 expression was more concentrated around the cytoplasm. Membrane accentuation of CK7 is already reported in renal carcinoma tissues [20] which supports the present findings. ISGF3 expression was highly elevated in low-grade ovarian cancer, which may limit the development of further cancer stages. However, notably ISGF3 $\gamma$ expression is reduced, which may promote the development of high-grade ovarian cancer. The present data showed experimental evidence which was previously reported with hypothetical definitions (reff).

In summary, the authors revealed the importance of ISGF3 expression as an anti-tumor agent which is expressed more in low-grade ovarian cancer in order to restrict the development of tumor. At the same time in high-grade ovarian cancer, its expression is notably downregulated which hinders anti-tumor activity.

\section{Acknowledgements}

This study was supported by Scientific research project of Hubei provincial health commission (No: WJ2019F017).

\section{Conflict of interest}

The authors declare no conflict of interest.

Submitted: July 02, 2019

Accepted: September 18, 2019

Published: October 15, 2020

\section{References}

[1] Torre L.A., Bray F., Siegel R.L., Ferlay J., Lortet-Tieulent J., Jemal A.: "Global cancer statistics, 2012". Ca. Cancer J. Clin., 2015, 65, 87-108.

[2] Siegel R.L., Miller K.D., Fedewa S.A., Ahnen D.J., Meester R.G.S., Barzi A., et al.: "Colorectal cancer statistics, 2017”. Ca. Cancer J. Clin., 2017, 67, 177-193.

[3] Marcus C.S., Maxwell G.L., Darcy K.M., Hamilton C.A., McGuire W.P.: "Current Approaches and Challenges in Managing and Monitoring Treatment Response in Ovarian Cancer". Journal of Cancer, 2014, 5, 25-30.

[4] Bowtell D.D., Böhm S., Ahmed A.A., Aspuria P., Bast R.C., Beral V., et al.: "Rethinking ovarian cancer II: reducing mortality from high-grade serous ovarian cancer". Nature Reviews Cancer, 2015 , $15,668-679$.

[5] Hansen J.M., Coleman R.L., Sood A.K.: "Targeting the tumour microenvironment in ovarian cancer". Eur. J. Cancer, 2016, 56, 131143.

[6] Coffelt S.B., de Visser K.E.: "Systemic inflammation: Cancer's long-distance reach to maximize metastasis". Oncoimmunology, 2016, 5, e1075694

[7] Kitamura T., Qian B., Pollard J.W.: "Immune cell promotion of metastasis". Nature Reviews Immunology, 2015, 15, 73-86.

[8] Svoboda M., Meshcheryakova A., Heinze G., Jaritz M., Pils D., Castillo-Tong D.C., et al.: "AIDAPOBEC-network reconstruction identifies pathways associated with survival in ovarian cancer". Bmc Genomics, 2016, 17.

[9] Hanahan D., Weinberg R.: "Hallmarks of Cancer: The Next Generation". Cell, 2011, 144, 646-674.

[10] Darnell J., Kerr I., Stark G.: “Jak-STAT pathways and transcriptional activation in response to IFNs and other extracellular signaling proteins". Science, 1994, 264, 1415-1421.

[11] Stark G., Darnell J.: "The JAK-STAT Pathway at Twenty". Immunity, 2012, 36, 503-514. 
[12] Huang Y., Jiang W., Wang Y., Zheng Y., Cong Q., Xu C.: “Enhanced efficacy and specificity of epithelial ovarian carcinogenesis by embedding a DMBA-coated cloth strip in the ovary of rat". $J$. Ovarian Res., 2012, 5, 21.

[13] Wauters C.C.A.P., Smedts F., Gerrits L.G.M., Bosman F.T. Ramaekers F.C.S.: "Keratins 7 and 20 as diagnostic markers of carcinomas metastatic to the ovary". Hum. Pathol., 1995, 26, 852-855.

[14] Berezowski K., Stastny J.F., Kornstein M.J.: "Cytokeratins 7 and 20 and carcinoembryonic antigen in ovarian and colonic carcinoma". Mod. Pathol., 1996, 9, 426-429.

[15] Adhikary T., Wortmann A., Finkernagel F., Lieber S., Nist A., Stiewe T., et al.: "Interferon signaling in ascites-associated macrophages is linked to a favorable clinical outcome in a subgroup of ovarian carcinoma patients". Bmc Genomics, 2017, 18,

[16] Borden E.C., Sen G.C., Uze G., Silverman R.H., Ransohoff R.M., Foster G.R., et al.: "Interferons at age 50: past, current and future impact on biomedicine". Nature Reviews Drug Discovery, 2007, 6, 975-990.

[17] Liao L., Liu Z.Z., Langbein L., Cai W., Cho E. A., Na J., et al:: "Multiple tumor suppressors regulate a HIF-dependent negative feedback loop via ISGF3 in human clear cell renal cancer". Elife., 2018, 7, e37925.

[18] Mevissen M., Häussler M., Szamel M., Emmendörffer A., ThunBattersby S., Löscher W.: "Complex effects of long-term $50 \mathrm{~Hz}$ magnetic field exposure in vivo on immune functions in female
Sprague-Dawley rats depend on duration of exposure". Bioelectromagnetics, 1998, 19, 259-270.

[19] Kriplani D., Patel M.: "Immunohistochemistry: A diagnostic aid in differentiating primary epithelial ovarian tumors and tumors metastatic to the ovary". South Asian Journal of Cancer, 2013, 2 , 254.

[20] Liu L., Qian J., Singh H., Meiers I., Zhou X., Bostwick D.G.: "Immunohistochemical analysis of chromophobe renal cell carcinoma, renal oncocytoma, and clear cell carcinoma: an optimal and practical panel for differential diagnosis". Arch. Pathol. Lab. Med., 2007, $131,1290-1297$.

Corresponding Author:

JING YANG, M.D.

Reproductive Medicine Center, Renmin Hospital of Wuhan University, Hubei Clinic Research Center for Assisted Reproductive Technology and Embryonic Development, Wuhan 430060 (China).

e-mail: JohnathonStevensmrr@yahoo.com 